\title{
Evolution studies of the CMS endcap calorimeter response and implications for the High-Luminosity LHC upgrade
}

\author{
Michael Planer ${ }^{* \dagger}$ \\ University of Notre Dame \\ 225 Nieuwland Science Hall, Notre Dame, IN 46556, USA \\ E-mail: mplanerecern.ch
}

\begin{abstract}
Calorimetry for the CMS detector is currently performed at the LHC with a lead tungstate crystal electromagnetic calorimeter (ECAL) and a brass/scintillator hadronic calorimeter (HCAL), both divided into barrel and endcap regions. High-Luminosity running at the LHC, which is planned for 2022 and beyond, will imply an order of magnitude increase in radiation levels and particle fluences with respect to the present LHC running conditions. The performance evolution of the CMS calorimeters indicates that an upgrade of the endcap calorimeters will be needed to ensure adequate performance during HL-LHC running. Results from LHC collision periods, beam tests and laboratory measurements are combined to predict the performance of the current detector at the HL-LHC and motivate the need for an upgrade of the endcap calorimeters.
\end{abstract}

Technology and Instrumentation in Particle Physics 2014,

2-6 June, 2014

Amsterdam, the Netherlands

\footnotetext{
${ }^{*}$ Speaker.

$\dagger$ on behalf of the CMS Collaboration
} 


\section{Introduction}

The Compact Muon Solenoid (CMS) [1] detector was originally designed to operate for about ten years, for LHC instantaneous/integrated luminosities of $1 \times 10^{34} \mathrm{~cm}^{-2} \mathrm{~s}^{-1}$ and $500 \mathrm{fb}^{-1}$ respectively. The High Luminosity LHC (HL-LHC) will provide an instantaneous luminosity of $5 \times 10^{34}$ $\mathrm{cm}^{-2} \mathrm{~s}^{-1}$; CMS will accumulate an integrated luminosity of $3000 \mathrm{fb}^{-1}$ by about 2035 [2]. In order to provide the same level of performance during the HL-LHC both the CMS electromagnetic and hadronic calorimeters will have to be upgraded.

\subsection{CMS endcap calorimeters}

TThe CMS electromagnetic calorimeter (ECAL) endcap is a homogeneous, hermetic, high granularity, and high resolution detector composed of PbWO4 scintillating crystals [1]. These crystals have a light yield of roughly 100 photons per $\mathrm{MeV}$ and a radiation length of $0.89 \mathrm{~cm}$. The endcap sections are composed of 14648 crystals and cover $1.48<|\eta|<3.0$ [1]. The CMS hadronic calorimeter (HCAL) endcap is a sampling calorimeter composed of alternating layers of brass absorber and plastic scintillating tiles [1]. The endcap portion covers $1.3<|\eta|<3$ with 19 layers [1].

\subsection{Motivation for endcap calorimeter upgrade}

Although the ECAL endcaps will perform well through the entire LHC operating period, the crystals will lose transparency from the high integrated luminosity of the HL-LHC; the high levels of radiation produce color centers in the crystals. The high instantaneous luminosity also poses several challenges, as described below, which an upgraded endcap electromagnetic calorimeter could better handle. The radiation damage in the HCAL endcap at the HL-LHC will cause transparency loss of the plastic scintillators necessitating an upgrade of the endcap hadronic calorimeter.

\section{ECAL endcap transparency loss}

The high integrated luminosity of the HL-LHC will cause transparency losses in the ECAL endcap which will severely impact the physics performance. Extrapolation from run 1 data, dedicated test beams, and simulations show that by the HL-LHC the ECAL endcap will need to be replaced with a new detector [2]. Figure 1a shows the relative signal response of the ECAL detector. A large part of the endcap will have less than $10 \%$ of the initial transparency, which directly impacts the constant term in the energy resolution. Figure $1 \mathrm{~b}$ shows the dependence of the mass resolution for Higgs boson decaying to 2 photons as a function of integrated luminosity. 
Figure 1

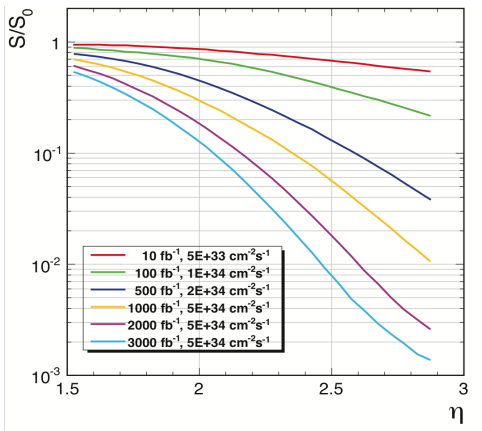

(a) Using response measurements from 2011 and 2012 ECAL operation and dedicated test beams, a model was developed to simulate the relative signal(S/S0) in the endcap region of ECAL. $500 \mathrm{fb}^{-1}$ corresponds to the beginning of the HL-LHC.

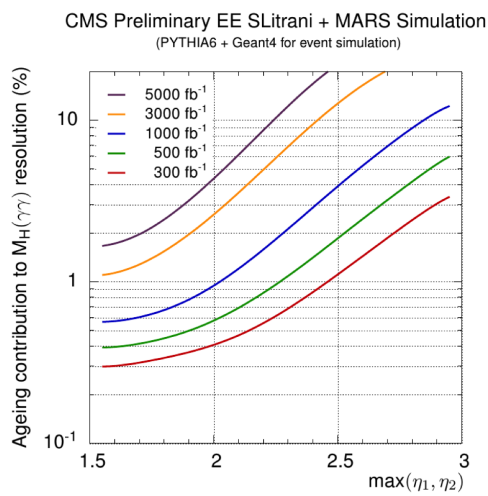

(b) Contribution to the Higgs mass width due to ECAL aging for the two photon decay channel. The mass resolution is calculated using $\mathrm{H} \rightarrow \gamma \gamma$ events generated with Pythia at center of mass energy of $14 \mathrm{TeV}$. The photon energy is smeared using test-beam energy resolution.

\section{ECAL pileup challenges}

In the high-pileup environment of the HL-LHC the current pulse shaping in ECAL is distorted by pileup events from previous bunch crossings. The pulse shape produced by the front-end electronics in ECAL is $42 \mathrm{~ns}$, while the bunch spacing is $25 \mathrm{~ns}$ at the HL-LHC. Energy deposited in a crystal from earlier bunch crossings can contaminate the signal. The contribution to the energy resolution in the ECAL endcaps from out-of-time pileup can be on the order of $10 \mathrm{~s}$ of $\mathrm{GeV}$ per crystal (Figure 3). The current ECAL endcap cluster size is roughly 25 crystals, which compounds this effect. By upgrading the ECAL endcap it is possible to mitigate this worsening of energy resolution due to out-of-time pileup.

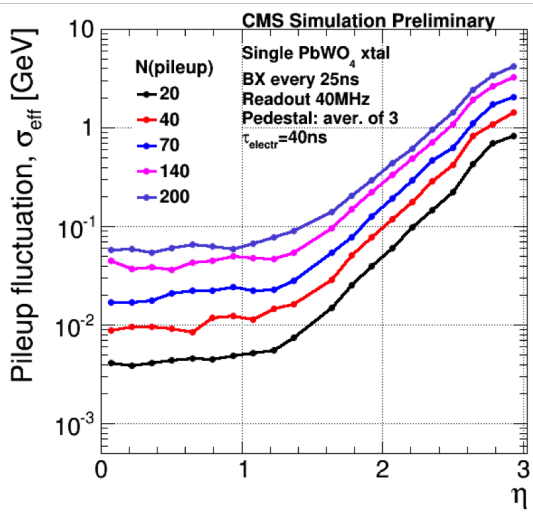

Figure 2: Average fluctuation of pileup energy deposit in a single ECAL crystal at each bunch crossing. This is, effectively, the noise due to pileup. 
Being able to improve the spike filtering at the trigger level will reduce the fake trigger rate for ECAL at the HL-LHC considerably. Upgrading the VFE will allow timing (currently used offline) to be improved and used at the trigger level to veto spikes. The full event readout at $40 \mathrm{MHz}$ will allow the shower shape variable (currently used offline) to be used at the trigger level. This allows the ECAL to minimize the effect of spikes at the trigger level using techniques developed offline in run 1.

\section{HCAL transparency loss}

The integrated luminosity of the HL-LHC will also severely reduce the transparency of the HCAL endcap tiles. At the start of the HL-LHC a region of the HCAL endcap will already be below 5\% of the nominal signal. By the end of the HL-LHC, a large fraction of the HCAL endcap will be significantly compromised.

Figure 3

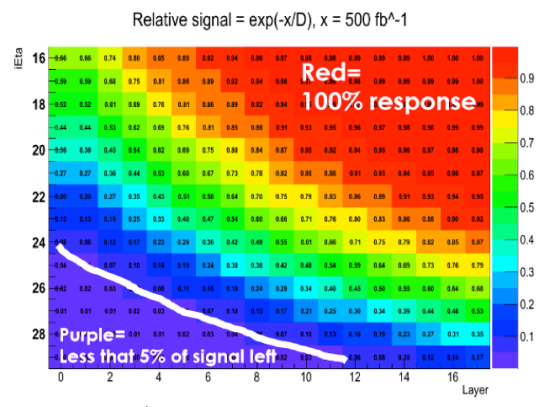

(a) At $500 \mathrm{fb}^{-1}$ performance of high- $\eta$ region of HE would be compromised.

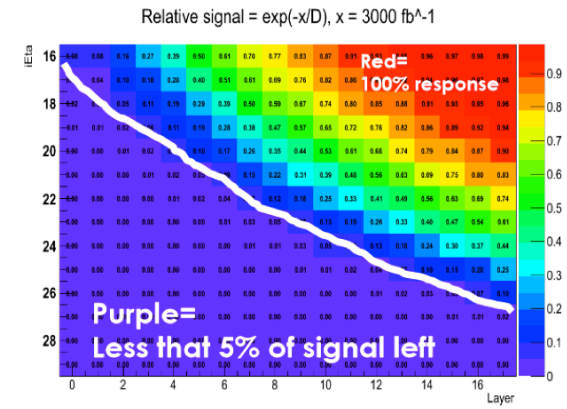

(b) At $3000 \mathrm{fb}^{-1}$ performance of entire HE would be compromised.

\section{Conclusion}

The decrease in light transmission of the ECAL endcap and the increased number of deposits in ECAL endcap due to high pileup during the operation of the HL-LHC severely decreases the physics performance of the current ECAL endcap. Similarly, the physics performance of the current HCAL endcap will be significantly degraded due to light transmission loss in the plastic scintillating tiles. Barrel ECAL and HCAL detectors will still function well, but the ECAL and HCAL endcaps must be replaced to ensure that CMS calorimetry during the HL-LHC will perform well.

\section{References}

[1] The CMS Collaboration,"The CMS experiment at the CERN LHC". JINST,3 (2008). S08004, doi:10.1088/1748-0221/3/08/S08004.

[2] F. Nessi Tedaldi,"The CMS experiment at the CERN LHC". 2012 IEEE Nuclear Science Symposium Conference also available as CMS-CR-2012/296 [arxiv.org/pdf/1211.3885v1.pdf]

[3] Adzic P et al., "Reconstruction of the signal amplitude of the CMS electromagnetic calorimeter". Eur. Phys. J.C 46 ,s1 (2006) 23-35 